\title{
Floral homeotic $C$ function genes repress specific $B$ function genes in the carpel whorl of the basal eudicot California poppy (Eschscholzia californica)
}

\author{
Aravinda L Yellina', Svetlana Orashakova', Sabrina Lange', Robert Erdmann', Jim Leebens-Mack², Annette Becker ${ }^{1 *}$
}

\begin{abstract}
Background: The floral homeotic C function gene AGAMOUS (AG) confers stamen and carpel identity and is involved in the regulation of floral meristem termination in Arabidopsis. Arabidopsis ag mutants show complete homeotic conversions of stamens into petals and carpels into sepals as well as indeterminacy of the floral meristem. Gene function analysis in model core eudicots and the monocots rice and maize suggest a conserved function for $A G$ homologs in angiosperms. At the same time gene phylogenies reveal a complex history of gene duplications and repeated subfunctionalization of paralogs.

Results: ESCAAG1 and ESCaAG2, duplicate AG homologs in the basal eudicot Eschscholzia californica show a high degree of similarity in sequence and expression, although ESCaAG2 expression is lower than ESCaAG1 expression. Functional studies employing virus-induced gene silencing (VIGS) demonstrate that knock down of EScaAG1 and 2 function leads to homeotic conversion of stamens into petaloid structures and defects in floral meristem termination. However, carpels are transformed into petaloid organs rather than sepaloid structures. We also show that a reduction of ESCAAG1 and ESCAAG2 expression leads to significantly increased expression of a subset of floral homeotic B genes.

Conclusions: This work presents expression and functional analysis of the two basal eudicot AG homologs. The reduction of ESCaAG1 and 2 functions results in the change of stamen to petal identity and a transformation of the central whorl organ identity from carpel into petal identity. Petal identity requires the presence of the floral homeotic B function and our results show that the expression of a subset of B function genes extends into the central whorl when the $C$ function is reduced. We propose a model for the evolution of $B$ function regulation by $C$ function suggesting that the mode of B function gene regulation found in Eschscholzia is ancestral and the Cindependent regulation as found in Arabidopsis is evolutionarily derived.
\end{abstract}

\section{Background}

Flowers are complex structures composed of vegetative and reproductive organs that are arranged in concentric whorls in most angiosperms. The vegetative floral organs, the sepals and the petals, develop in the outer whorls while the inner whorls are composed of the pollen-bearing stamens and in the center carpels enclose the ovules. The carpels are the last organs formed in the flower and the floral meristem is consumed in the process of carpel development [1]. As described by the

\footnotetext{
* Correspondence: annette.becker@uni-bremen.de

'University of Bremen, Fachbereich 02 Biology/Chemistry, Evolutionary

Developmental Genetics Group Leobener Str., UFT, 28359 Bremen, Germany Full list of author information is available at the end of the article
}

ABCDE model, floral homeotic transcription factors act in a combinatorial fashion to determine the organ identity primordia for the four distinct whorls: A + E class genes specify sepal identity; $\mathrm{A}+\mathrm{B}+\mathrm{E}$ class genes act together to determine petal identity; $\mathrm{B}+\mathrm{C}+\mathrm{E}$ class genes specify stamen identity; $\mathrm{C}+\mathrm{E}$ class genes together define carpel identity, and $C+D+E$ class genes specify ovule identity $[2,3]$. Most of these homeotic functions are performed by members of the MADS-box gene transcription factor family. AGAMOUS (AG), a C class gene in Arabidopsis is necessary for specification and development of stamen and carpals, and floral meristem determinacy [4]. The flowers of the strong $a g-1$ mutant shows complete homeotic conversions of stamens into

\section{C) Biomed Central}


petals and carpels into sepals and a recurrence of these perianth organs in a irregular phyllotaxy [5].

Members of the $A G$ subfamily of MADS box genes have been identified in all major clades of seed plants but not in more basal, seed-free lineages indicating that the $A G$ clade originated around 300 to 400 million years ago in the common ancestor of gymnosperms and angiosperms. In gymnosperm species, $A G$ orthologs were found to be expressed in male and female reproductive cones, which is reminiscent of the angiosperm expression in stamens and carpels [6-8]. Gene family phylogenies reveal several duplication events within $A G$ clade of MADS box genes (Figure $1[9,10]$ ). The first duplication event at the base of the angiosperm lineage led to the origins of the SEEDSTICK and $A G$ clades including ovule specific $\mathrm{D}$ class genes and the carpel and stamen specifying $C$ class genes, respectively [10]. A more recent duplication in the C-lineage gave rise to the PLENA clade and euAG clade, the

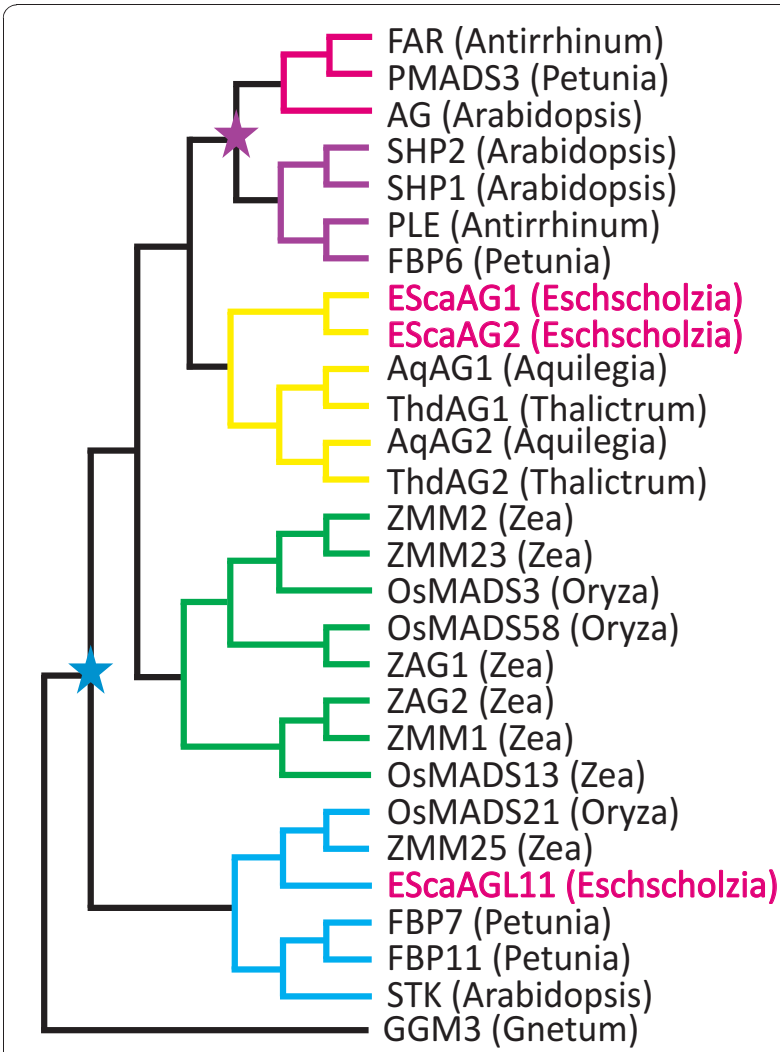

Figure 1 Simplified phylogeny indicating duplication events of the AG lineage in angiosperms based on Zahn et al., 2006 [18]. Red branches denote euAG lineage genes, purple branches the PLE lineage genes, yellow branches symbolize the basal eudicot lineage, green branches denote the monocot $C$ class genes and blue branches denote D class genes. GGM3 represents the gymnosperm lineage of $A G$ homologs. The California poppy genes are marked in red letters. The blue star symbolizes the C/D duplication event and the purple star indicates the EuAG/PLE duplication. former containing the Arabidopsis SHATTERPROOF1 and 2 genes (SHP1 and 2), the latter AG. This duplication occurred after the ranunculids (basal eudicots in the order Ranunculales) diverged from the lineage leading to the core eudicots $[9,11]$.

The Arabidopsis members of the PLENA clade, SHP1 and 2 are required for dehiscence zone differentiation in the fruit and consequently for pod shattering [12,13]. Interestingly, PLENA itself, a gene in Antirrhinum majus, is functionally more similar to $A G$ than SHP1 and 2, and FARINELLI (FAR), the Antirrhinum AG ortholog is required for pollen development. Both $F A R$ and PLENA are necessary for floral meristem determinacy in Antirrhinum [14,15].

Gene duplications and subfunctionalization have also occurred in C-lineage of monocots, but independently of the eudicot duplications (Figure 1). ZAG1 from maize is required for floral meristem determinacy and $Z M M 2$ is involved in stamen and carpel identity [16]. The rice homologs OSMADS3 and OSMADS58 share common functions, but also show a degree of subfunctionalization. While OSMADS3 plays a major role in stamen and a minor role in carpel identity, OSMADS58 has a strong influence on carpel identity and floral meristem determination [17]. Independent duplications of $A G$ homologs have been inferred for other flowering plant lineages, but functional analyses of duplicated AG homologs are sorely lacking outside of model core eudicot and grass species.

Here we report functional data of the $A G$ homologs of the basal eudicot Eschscholzia californica (California poppy, Papaveraceae) that belongs to Ranunculales, a basal eudicot order. Basal eudicots are a sister grade leading to the more diverse core eudicot clade. Investigation of species in this grade can shed light on the divergence of monocots and eudicots and events that may have promoted diversification within the core eudicots.

Two AG homologs, EScaAG1 and EScaAG2, and a D lineage homolog, EScaAGL11, have been identified in E. californica. EScaAG1 and EScaAG2 show similar expression patterns, but EScaAG1 is expressed at a much higher level than EScaAG2 [18]. The expression patterns of both genes resembles that of AGAMOUS (AG) in Arabidopsis except that the Eschscholzia poppy $A G$ orthologs are expressed earlier in the floral meristem $[18,19]$.

This work presents an experimental investigation of the EScaAG1 and EScaAG2 gene function employing VIGS to manipulate transcript concentrations. We map the expression of both genes in more detail than previously published and demonstrate that the down regulation of $\mathrm{C}$ function genes in E. californica leads to an induction of some floral homeotic B genes in the fourth floral whorl. 


\section{Results}

ESCaAG1 and ESCaAG2 are very similar in sequence and expressed differentially

The two AG homologues of E. californica, EScaAG1 and EScaAG2, share $66.6 \%$ and $61.1 \%$ amino acid sequence identity to $A G$ of Arabidopsis, respectively. These paralogs are very similar throughout the open reading frame and in the 5'untranslated region (UTR) with $75 \%$ identity at the nucleotide level and about $81.7 \%$ at the amino acid level (Additional file 1). When the two paralogues are compared along their UTR and open reading frame, the EScaAG2 nucleotide sequence shows a $45 \mathrm{bp}$ insertion and $14 \mathrm{bp}$ deletion in the 5' UTR and a 10 bp deletion in the 3' part of coding region of EScaAG1 (data not shown).

Quantitative Reverse Transcriptase (RT)-PCR was carried out on cDNA derived from floral organs at anthesis, young fruits, leaves, and buds of different developmental stages to learn more about the differential expression of EScaAG1 and EScaAG2 (Figure 2A). Both genes are expressed in the reproductive organs of the flower, in young fruits and in all tested stages of flower development. EScaAG1 and EScaAG2 are expressed in sepals, petals, and leaves at extremely low levels. EScaAG1 is highly expressed in stamens, carpels, young fruits and later stages of flower development. EScaAG2 is generally expressed at a lower level than EScaAG1 with the exception of stamen, where its expression is about $1.5 \times$ higher than that of EScaAG1. In young fruits and during bud development, EScaAG2 transcript abundance is very low in comparison to EScaAG1.

The spatial expression patterns of EScaAG1 and 2 were additionally analyzed through in situ hybridizations to obtain a more detailed picture of the expression domains. However, as the open reading frames and UTR's of EScAG1 and EScaAG2 are highly similar, we were unable to generate probes that could discriminate between both genes. As a consequence, in situ hybridization patterns were nearly identical for these genes. The only difference between the in situ hybridization patterns was a much lower level of expression for EScaAG2 (data not shown). In the following section, we refer to the composite expression of EScaAG1 and EScaAG2 as EScaAG1/2 expression patterns.

EScaAG1/2 gene expression was first observed in the stage 2 bud before the gynoecium initiates and was visible as lateral domains in a few cells in the floral meristem where later the stamen primordia are initiated (Figure 2B). In a stage 4 bud, the expression expands uniformly in the floral meristem but is excluded from the central primordium where later the gynoecium arises (Figure 2C). By late stage 4, EScaAG1/2 expression becomes restricted to the boundaries between the stamen anlagen with weak expression at the tip in the floral meristem just before gynoecium initiates (Figure $2 \mathrm{D})$. In stage 6 , strong expression is found in the region adjacent to the placenta, the apical part of the medial carpel wall and in the stamens (Figure 2E). Later in late stage 6, EScaAG1/2 expression is restricted to the adaxial side of the gynoecium and in the stamens (Figure $2 \mathrm{~F})$. In transverse sections of the developing flower bud, EScaAG expression is confined to the apical part of the ovules but not in the placenta. In later stages of ovule development, the EScaAG1/2 expression is stronger on the adaxial than on the abaxial side (Figure 2G, H, I). In summary, EScaAG1/2 genes are expressed during floral meristem initiation at stage 2 , during early development of stamen and carpel primordia and later in the developing stamens and ovules.

\section{ESCaAG1 and ESCaAG2 confer stamen identity}

Virus induced gene silencing (VIGS ) was employed to investigate the functions of EScaAG1 and EScaAG2 during flower development. This method allows transient down-regulation of gene expression via modified plant viruses, in our case the Tobacco Rattle Virus (TRV). The E. californica flower is composed of a single sepal occupying the first floral whorl, two whorls of four petals and a varying number of stamen whorls ranging from four to eight. The inner floral whorl produces a bicarpellate gynoecium (Figure 3A) [20]. Overall, the phenotypic effects of the EScaAG1 and EScaAG2 VIGS were restricted to flowers. Treatment plants exhibited a loss of stamen identity, homeotic conversion of stamens into petals, and a loss of carpel characteristics. Additionally, EScaAG1 and 2 VIGS results in a loss of floral meristem termination. None of the analyzed pTRV2-E (mock treatment, treated with the empty pTRV2 vector) treated or untreated plants showed homeotic conversions or signs of loss of floral meristem termination, and the vegetative habit also did not show any deviations from untreated plants (Table 1 and [21]).

In total, 120 plants were infected with pTRV2EScaAG1, 120 plants with pTRV2-EScaAG2, another 120 plants were inoculated with pTRV2-EScaAG1/2, and 12 plants were infected with pTRV2-E as a mock control. The first three flowers of each plant were analyzed because the frequency of phenotype decreases in the later formed flowers [21]. The phenotype scores for each treatment are summarized in Table 1: 239 flowers of plants infected with pTRV2-EScaAG1 were analyzed, of which 122 flowers (51.0\%) showed homeotic conversion in the third and fourth whorl floral organs. Of these 122 flowers, $4.5 \%$ showed homeotic conversion of all stamens into petal-like organs (Figure 3B). A total of 209 flowers of plants infected with pTRV2-EScaAG2 were observed, and of these 118 flowers (56.4\%) showed 


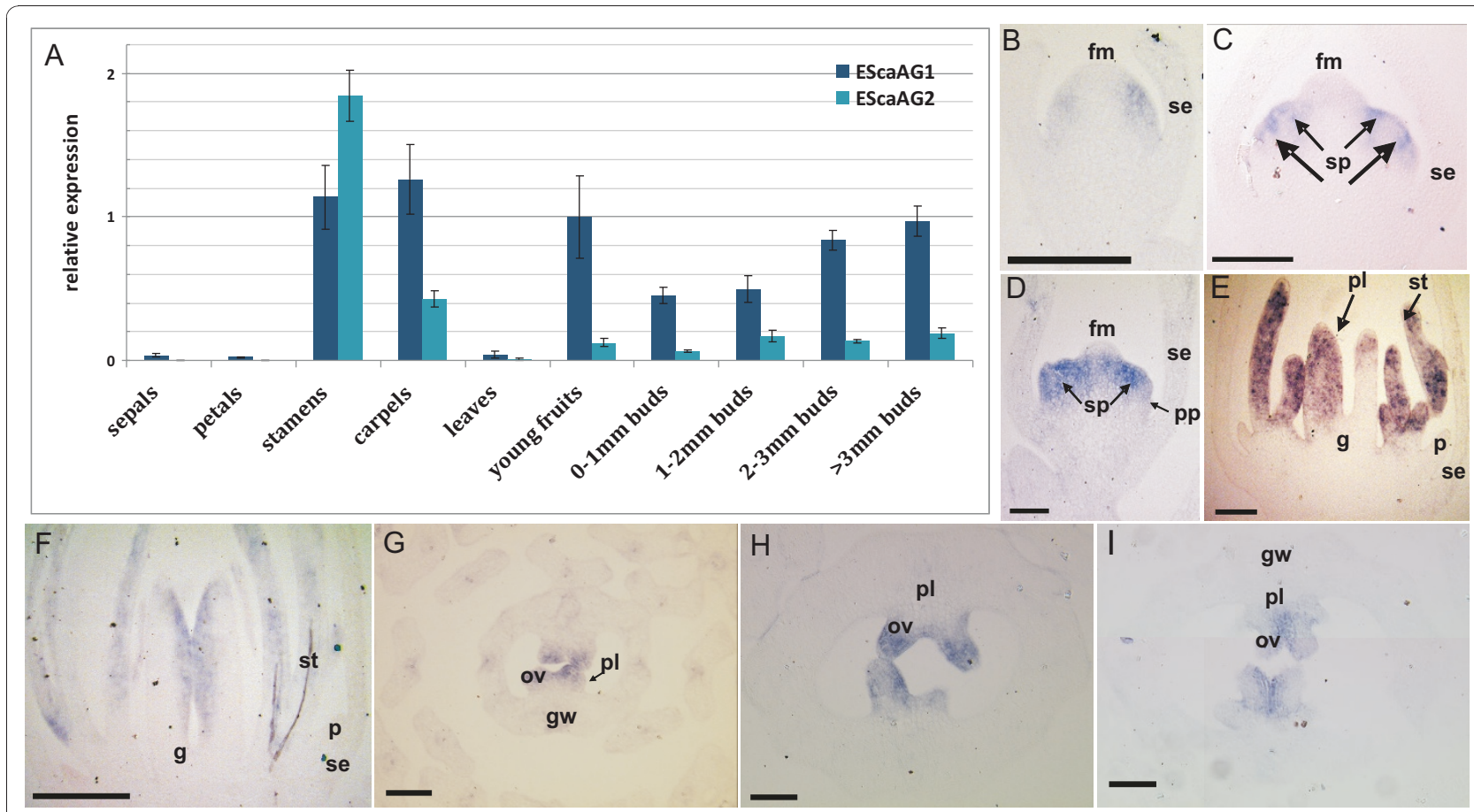

Figure 2 Expression analysis of ESCAAG1 and ESCaAG2 in flowers of untreated plants shown by quantitative RT-PCR and in situ hybridization. (A) Q-PCR based relative expression analysis of ESCaAG1 and ESCaAG2 in E. californica. Actin and GAPDH were used as reference genes. (B) to (I) in situ hybridization pattern of the ESCaAG genes using an ESCaAG1 probe. (B) Longitudinal section of a bud in stage 2. (C) Longitudinal sections of a bud in stage 3. (D) Longitudinal sections of a bud in stage 4. (E) Longitudinal section of a bud in late stage 6. (F) Longitudinal section of a bud in stage 7. (G) Transverse section of a bud in stage 7. (H) Transverse section of a bud in stage 8. (I) Transverse section of a bud in stage 9. All scale bars $=100 \mu \mathrm{m}$, abbreviations: fm, floral meristem; g, gynoecium; gw, gynoecium wall; ov, ovule; p, petal; pl, placenta; pp, petal primordium; se, sepal; sp, stamen primordium; st, stamen.

homeotic transformation of stamens and carpels. Of all flowers developing a silencing related phenotype, 15\% exhibited complete homeotic transformation of all stamens into petal-like organs (Figure 3C). Of the 261 flowers of plants infected with pTRV2-EScaAG1/AG2, 174 flowers $(66.6 \%)$ showed homeotic transformation of stamens and carpels and 15\% of the latter exhibited complete homeotic transformation of all stamens into petal-like organs (Figure 3D-H, Table 1).

Interestingly, EScaAG1 and 2 VIGS-treated plants exhibited conversion of stamen to petaloid organs in different stamen whorls (Table 1). Focusing on plants infected with pTRV2-EScaAG1, 64 flowers (95.5\% of the flowers with homeotic conversion in the third whorl) showed partial homeotic transformation of only the outer whorls of stamens into petaloid organs (Figure 3I), while the inner stamen whorls maintained a wild type appearance. In contrast, 45 flowers $(84.9 \%$ of the flowers with homeotic conversions in the third whorl), from plants infected with pTRV2-EScaAG2 showed homeotic conversion of only the inner stamen whorls to petaloid organs (Figure 3J). Plants infected with pTRV2EScaAG1/2 exhibited composite phenotypes: 96 flowers (84.9\% of the flower with homeotic conversion in the third whorl) exhibited partial homeotic conversion of outermost and innermost whorls while retaining wild type stamen morphology in the central stamen whorls (Figure 3K). Homeotic transformations of stamens into petaloid organs occurred in various degrees as we observed phenotypes ranging from complete petal-like organs (Figure 3F) to mosaic staminoid-petaloid structures (Figure 3K).

Histological transverse sections of EScaAG1 and 2 VIGS-treated plants reveal further details of the homeotic conversions of stamens and gynoecia (Figure 3L, M). In comparison to pTRV2-E treated plants (Figure 3M), the connective of the stamens in the silenced plants is elongated when compared to untreated plants and the theca contain three pollen sacs in a few cases rather than two as seen in untreated plants. The number of vascular bundles in the connective is also increased from one in untreated to five in stamens of VIGS treated plants. Additionally, the gynoecium in the center of the flower of VIGS-treated plants is composed of two fused parts reminiscent of petals. A solid ovary wall is missing in the VIGS-treated plants as well as lateral differentiation of the ovary wall, such as a placenta or ovules (Figure 3L, M). 

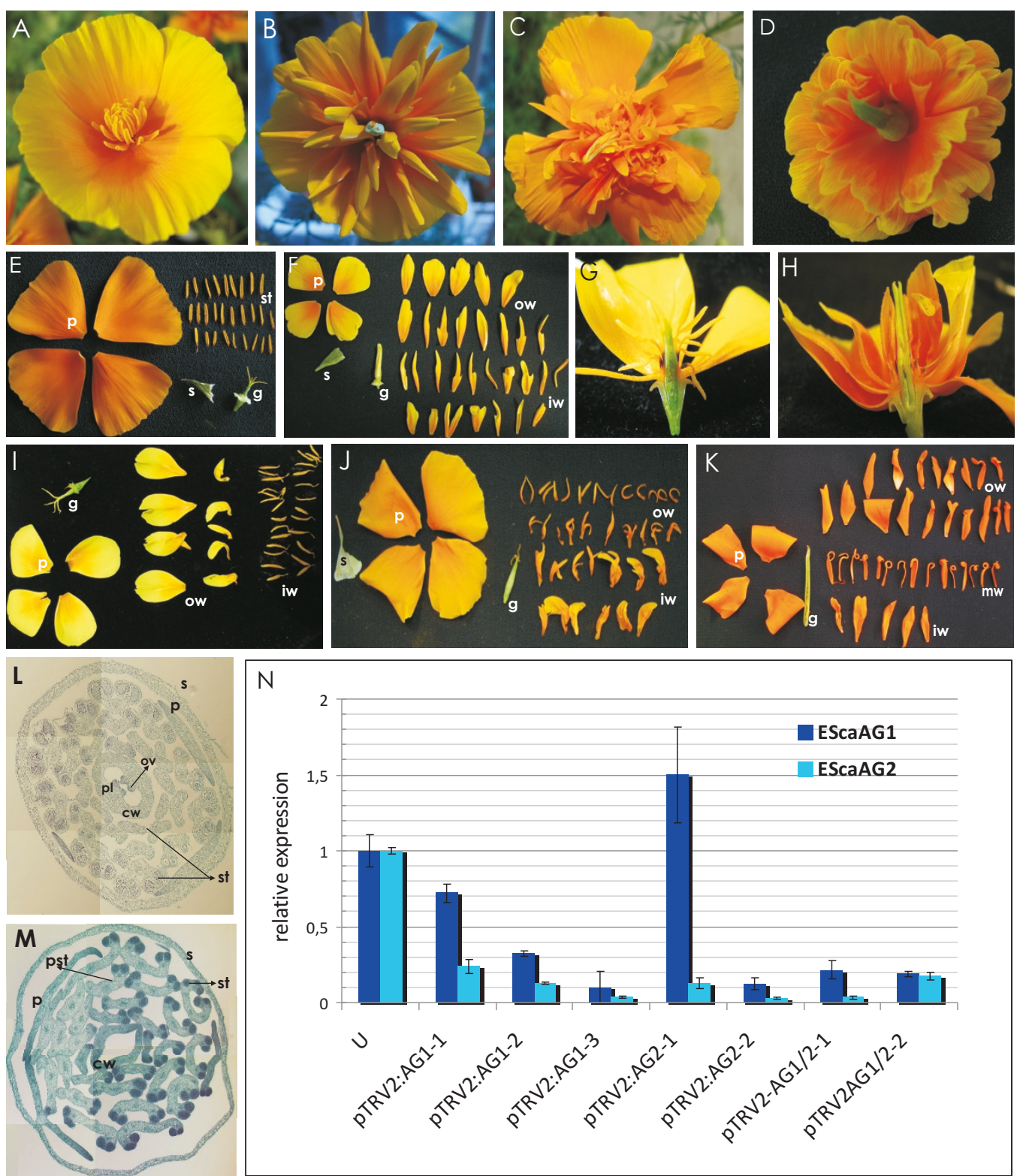

Figure 3 Phenotypes of plants treated with pTRV1 and pTRV2-EScaAG1, pTRV2-EScaAG2, or pTRV2-EScaAG1/2 and expression analysis of the VIGS treated plants. (A) Wild type phenotype of an E. californica flower treated with pTRV2-E. (B) Phenotype of an EScaAG1 VIGS treated plant showing full homeotic conversions of stamens into petals. (C) Phenotype of an EScaAG2 VIGS treated plant showing full homeotic conversions of stamens and carpels into petal-like structures. (D) Phenotype of a flower silenced for EScaAG1/2 showing homeotic conversions of stamens into petals. (E) A mock treated plant with disassembled floral organs. (F) Disassembled flower of a plant treated with pTRV2-EScaAG1/2 showing homeotic conversions of stamens into petals. The same phenotype was also achieved with plants silenced for ESCaAG1 or EScaAG2 individually. (G) Transverse hand section of a flower from a mock treated plant. (H) Transverse hand section of a flower from a plant silenced for EScaAG1/2 showing homeotic conversions of stamens into petals. (I) Disassembled flower of a plant silenced for EScaAG1 showing partial homeotic conversions of only the outer whorl stamens. (J) Disassembled flower of a plant treated with pTRV2-EScaAG2 showing that the inner whorl of stamens is converted into petal-like structures. (K) Disassembled flower of a plant treated with pTRV2-EScaAG1/2 exhibiting homeotic conversions of the innermost and outermost stamens whorls into petals while the middle whorls show mild deviation from wild type stamens while the center whorl stamens remain more stamen-like. (L) Transverse section of a flower of an untreated plant. (M) Transverse section of a flower from an ESCaAGI VIGS treated plant showing homeotic conversion of stamens into petals, petal-stamen mosaic structures, malformed stamens and a gynoecium lacking tissue differentiation, ovules, and placenta. (N) Real-Time PCR analysis of the first bud of individual $E$. californica plants treated with VIGS and untreated (U). Plants were treated with pTRV2-EScaAG1 are abbreviated as VIGS AG1, plants treated with PTRV2-ESCaAG2 as VIGS AG2. Numbers below indicated individual plants and the relative expression level of ESCaAG1 and EScaAG2 in untreated plants was set to 1. Abbreviations: cw, carpel wall; ov, ovule; p, petal; pl, placenta; se, sepal; pst, petaloid stamens; st, stamen. 
Table 1 Overview of the observed phenotypes of EScaAG VIGS in California poppy

\begin{tabular}{|c|c|c|c|c|c|}
\hline & Phenotypes observed & $\begin{array}{l}\text { PTRV1/ } \\
\text { pTRV2-E }\end{array}$ & $\begin{array}{l}\text { pTRV1/pTRV2- } \\
\text { EScaAG1 }\end{array}$ & $\begin{array}{l}\text { pTRV1/pTRV2- } \\
\text { EScaAG2 }\end{array}$ & $\begin{array}{l}\text { pTRV1/pTRV2- } \\
\text { EScaAG1+2 }\end{array}$ \\
\hline 1 & No. of inoculated plants & 12 & 120 & 120 & 120 \\
\hline 2 & No. of analyzed flowers & 36 & 239 & 209 & 261 \\
\hline 3 & No. of flowers showing phenotype in the third and fourth whorls & 0 & $122(51.0 \%)$ & $118(56.4 \%)$ & $174(66.6 \%)$ \\
\hline 3.1 & No. of flowers with homeotic conversions in the stamens & 0 & $67(54.9 \%)$ & $53(44.9 \%)$ & $113(64.9 \%)$ \\
\hline 3.1 .1 & No. of flowers with transformation of all the stamens into petals & 0 & $3(4.4 \%)$ & $8(15 \%)$ & $17(15 \%)$ \\
\hline 3.1 .2 & $\begin{array}{l}\text { No. of flowers showing only outer stamen whorls converted into } \\
\text { petaloid organs }\end{array}$ & 0 & $64(95.5 \%)$ & 0 & 0 \\
\hline 3.1 .3 & $\begin{array}{l}\text { No. of flowers showing only inner stamen whorls converted into } \\
\text { petaloid organs }\end{array}$ & 0 & 0 & $45(84.9 \%)$ & 0 \\
\hline 3.1 .4 & $\begin{array}{l}\text { No. of flowers showing only outer and inner stamen whorls } \\
\text { converted into petaloid organs }\end{array}$ & 0 & 0 & 0 & $96(84.9 \%)$ \\
\hline 3.2 & No. of flowers with alterations in the carpels & 0 & $27(22.1 \%)$ & $31(26.2 \%)$ & $40(22.9 \%)$ \\
\hline 3.2 .1 & No. of flowers with flattened green gynoecium & 0 & $23(85.1 \%)$ & $26(83.8 \%)$ & $32(80 \%)$ \\
\hline 3.2 .2 & No. of flowers with an orange pigmented gynoecium & 0 & $4(17.3 \%)$ & $5(16.1 \%)$ & $8(20 \%)$ \\
\hline 3.3 & $\begin{array}{l}\text { No. of flowers showing defects in the floral meristem } \\
\text { termination }\end{array}$ & 0 & $62(50.8 \%)$ & $80(67.7 \%)$ & $110(63.2 \%)$ \\
\hline
\end{tabular}

The strength of the observed phenotypes was correlated with the degree of reduction in EScaAG1 and 2 transcript levels as measured by Q-PCR. The first floral bud (size 1 to $3 \mathrm{~mm}$ in diameter) of randomly selected plants treated with the EScaAG1, EScaAG2, and EScaAG1/2 VIGS vectors was collected and correlated with the phenotype of the next formed flower. In $99 \%$ of the cases $(n=414)$ we observed that when the secondarily formed flower showed a phenotype, the first flower exhibited a phenotype as well. This consistent pattern allowed us to predict the phenotype of the first bud used for quantitative RT-PCR based on the second flower's phenotype (see also [21,22]). The changes in EScaAG1 and EScaAG2 expression in the first buds (1 to $3 \mathrm{~mm}$ bud diameter) of individual VIGS treated plants are documented in Figure $3 \mathrm{~N}$. Targeted silencing of individual EScaAG genes was not achieved, suggesting that the overlap in observed phenotypes result from a reduction of expression of both $A G$ paralogs. Irrespective of the silencing vector used, EScaAG1 expression was generally reduced from $70 \%$ to $10 \%$ of its wild type expression and EScaAG2 expression was reduced from $25 \%$ to less than $5 \%$. The use of the pTRV2-EScaAG1/2 vector resulted in similar reductions in expression levels for both genes. While six plants show silencing of both, EScaAG1 and EScaAG2, one plant (pTRV2:AG2-1) treated with EScaAG2-targeted VIGS exhibited reduction of EScaAG2 expression but increased EScaAG1 expression relative to untreated plants, demonstrating the variability of VIGS experiments. However, we were able to show a significant reduction of expression in six of seven randomly analyzed buds from individual plants.
VIGS of C-function genes results in homeotic conversions of carpels into petal-like organs

In addition to homeotic conversions of stamens into petal-like structures in plants infected with pTRV2EScaAG1 and pTRV2-EScaAG2, we observed changes to the gynoecium morphology. The gynoecia of untreated plants develop as round green cylinders and consist of two fused carpels. This cylinder-like structure was disturbed in EScaAG1 and EScaAG2 VIGS-treated plants and the gynoecia of the VIGS treated plants were transformed either into (i) flattened green structures lacking ovules in some cases (Figure 4A Table 1) or (ii) flattened organs showing petal characteristics such as orange pigmentation and petal-like epidermal surface structure (Figure 4B Table 1). The latter was empty (Figure 4A, B) or contained additional floral organs (Figure $4 \mathrm{~F}$ Table 1).

In order to determine whether the petal-like pigmentation of the gynoecium was associated with a change in cell surface morphology we conducted Scanning Electron Microscopy (SEM) analysis of the carpel whorl. In the wild type, the carpel surface is composed of small compact cells interrupted by stomatal cells (Figure 4C) and the petal surface is composed of long and narrow cells arranged in a parallel manner (Figure 3D) [20]. SEM micrographs of an orange-pigmented gynoecium reveal a mosaic pattern of tubular petal-like cells next to small compact cells typical for a carpel surface scattered with stomata (Figure 3E). This indicates that the gynoecia of EScaAG1 and EScaAG2 VIGS treated plants not only show a partially petal-like pigmentation but have also acquired petal-like cell surface characteristics, 
supporting the hypothesis that these gynoecia are partially transformed into petal-like organs.

Treating poppy plants with EScaAG1 and 2 VIGS not only resulted in the loss of stamen and carpel characteristics but also in the addition of petal organ identity to the carpel whorl. We tested the hypothesisis that the expression domains of floral homeotic B genes was extended to the central gynoecium whorl in EScaAG1 and 2 VIGS treated plants using real-time PCR to assess expression of the three poppy floral homeotic B class genes EScaDEF1, EScaDEF2, and EScaGLO at anthesis and pre-anthesis (Figure 4G, H). B and C gene expression in untreated gynoecia was also characterized (Figure 4D). As expected EScaAG1 as well as EScaAG2 were expressed in gynoecia before and at anthesis. Surprisingly, the class B gene ortholog, EScaDEF1 was expressed in gynoecia at a comparatively high level, although expression levels of two other B-class genes EScaDEF2 and EScaGLO were hardly detectable. Next, the expression of class $\mathrm{B}$ and $\mathrm{C}$ genes was recorded in the gynoecia of VIGS treated plants (Figure 4G). The relative expression of all analyzed genes was normalized by setting levels to one in gynoecia of untreated plants before anthesis. In the gynoecia of VIGS treated plants (Figure $4 \mathrm{H}$ ), expression of EScaAG1 was reduced to $50 \%$ and even $20 \%$ in the gynoecia of VIGS treated plants and expression of EScaAG2 was reduced in most gynoecia as well. VIGS treatments had no impact on ESca$D E F 1$ expression in the gynoecia. However, the expression of EScaDEF2 was drastically increased between 5.8-fold and 17.7-fold relative to expression in untreated gynoecia. Transcript abundance of EScaGLO, also increased significantly upon silencing of $\mathrm{C}$ function genes by 2.2 to 5.7 times in the EScaAG1 and EScaAG2 VIGS treated plants. These expression analyses indicate that in central whorl organs with reduced expression of $\mathrm{C}$ function genes, two B function genes EScaDEF2 and EScaGLO were expressed at significantly higher level in EScaAG1 and EScaAG2 VIGS treated than in untreated or mock treated plants.

For the Arabidopsis B proteins APETALA3 (AP3) and PISTILLATA (PI) it was shown that their homeotic function requires the formation of AP3-PI heterodimers [23]. EScaDEF2 is an AP3 homolog while EScaGLO is the PI homolog [24] and simultaneous upregulation of the AP3 and PI orthologs in poppy suggests that they might form heterodimers in the central whorl of $\mathrm{C}$ function silenced flowers and cause the observed homeotic gynoecium-petal conversions.

\section{ESCaAG1 and ESCaAG2 are involved in the regulation of floral meristem termination}

The flowers of the plants treated with EScaAG1 and EScaAG2 VIGS showed not only homeotic conversions of stamens into petaloid organs, petal-like features in the central whorl, and a reduction in ovule number, but also signs of prolonged floral meristem activity. All treated plants showed increases in floral organ number in the stamen and central whorls. Moreover, flowers exhibiting a strong silencing phenotype showed ectopic structure enclosed inside the gynoecium whorl ranging from carpel like leaves to additional gynoecia and ectopic flowers (Figure 4F).

Interestingly, we observed a significant increase in stamen number in the weaker floral phenotypes characterized by no obvious homeotic organ conversions (Table 2). Untreated plants produced 26.2 stamens per flower on average, EScaAG1 VIGS-treated plants without any homeotic conversions developed 29 stamens per flower, EScaAG2 VIGS-treated produced 28.2, and plants treated simultaneously with $E S c a A G 1 / 2$ produced 28.6 stamens on average. This suggests that whereas a mild reduction in EScaAG1 and EScaAG2 expression may not affect floral organ identity any reduction in expression can induce an increase in stamen number.

\section{Discussion}

This study is the first functional analysis of floral homeotic $\mathrm{C}$ function genes in a basal eudicot. We employed VIGS to transiently down-regulate EScaAG1 and EScaAG2 in E. californica and observed homeotic conversions of stamens into petals, reduced floral meristem termination, and transformation of the gynoecium into petal-like structures. EScaAG2 is expressed at lower levels (also observed by [18]) but despite the reduced expression of EScaAG2, molecular evolutionary analyses failed to detect evidence of reduced evolutionary constraint (see below).

The two AG paralogs of E. californica, EScaAG1 and EScaAG2 are quite similar on both protein and nucleotide level including the 5'UTR region indicating that they are duplicates. Generally it is hypothesized, that duplicated genes will not persist over evolutionary time unless sub-, or neofunctionalization results in functional divergence [25-27]. EScaAG1 and EScaAG2 share about $81.7 \%$ sequence similarity in the open reading frame and are $75.5 \%$ identical when the 5'UTR is included. The origin of these paralogs may be associated with an ancient whole genome duplication event that has been inferred on the lineage leading to Eschscholzia [28]. Using a penalized likelihood approach [29] we estimated an age of 51 million years for the EScaAG duplication. This divergence time was obtained using a maximum likelihood tree for the $A G$ subfamily [30] calibrated with taxon ages reported in [31].

No evidence of reduced constraint on EScaAG2 was inferred from analysis of the ratio of nonsynonymous to synonymous nucleotide substitutions on the branch 

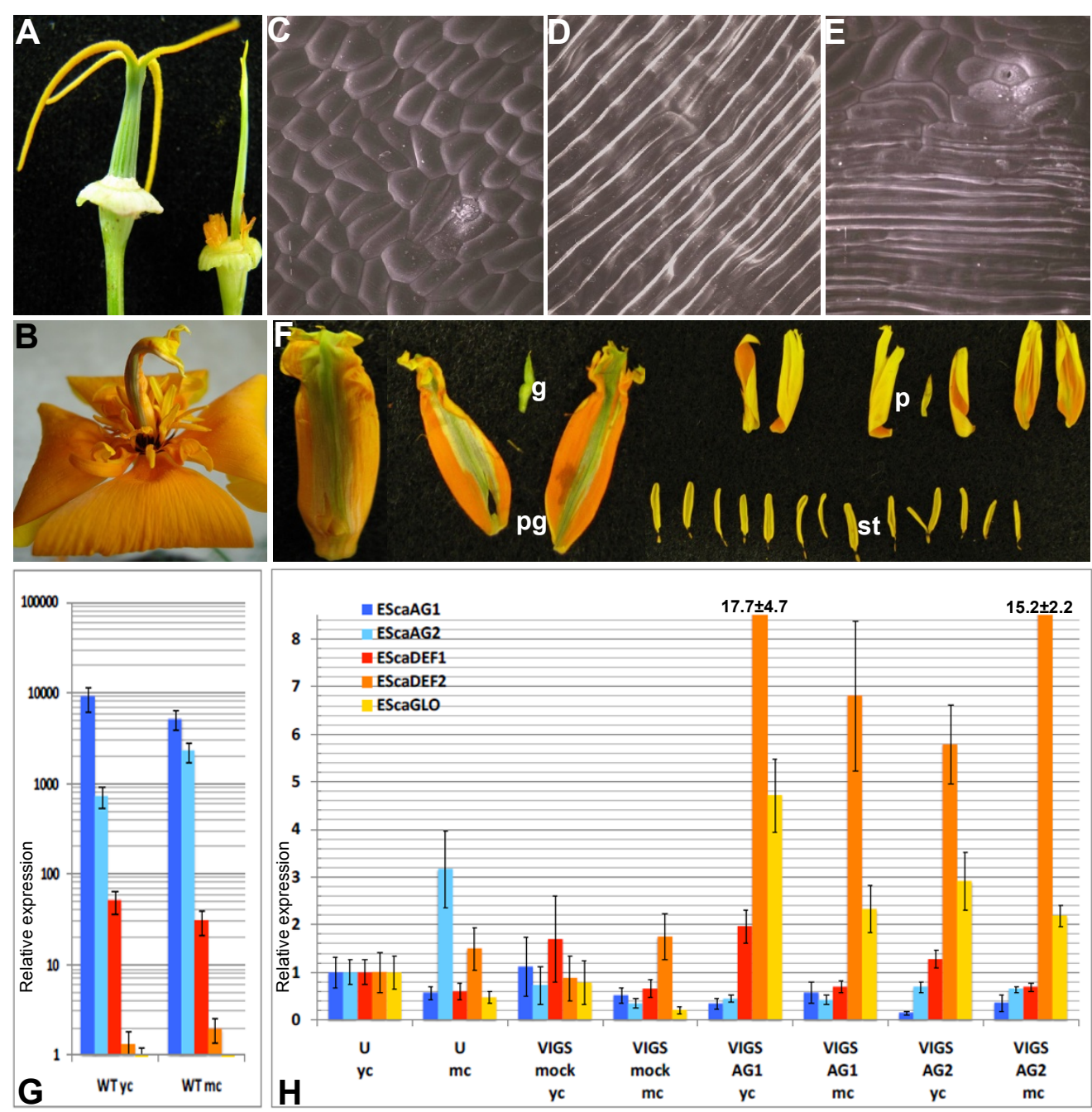

Figure 4 Carpel whorl phenotype of ESCaAG silenced plants and expression analysis of floral homeotic genes. (A) Gynoecium of an untreated plant (left) and of a plant silenced for ESCaAG2 (right) (B) Flat orange gynoecium without ovules of a plant treated with pTRV2ESCaAG1. (C) Scanning electron micrograph (SEM) of the wild type gynoecium surface structure. (D) SEM of a wild type petal surface structure. (E) SEM of the central floral whorl organ of a plant treated with pTRV2-ESCaAG1 showing a mix of petal and gynoecium surface structures. (F) The central whorl floral organ (pg, for petaloid gynoecium) of a plant treated with pTRV2-EScaAG2 showing petaloid and carpeloid features as well a lack of ovules. This organ encloses an ectopic flower consisting of a remnant gynoecium ( $\mathrm{g}$ ), petals (p) and stamens (st). (G) Relative expression of class B and C genes in young carpels before anthesis and mature carpels at anthesis of untreated plants, (H) Real-Time RT-PCR expression analysis of ESCaAG1, ESCaAG2, ESCaDEF1, ESCaDEF2, and ESCaGLO in the gynoecia of VIGS treated plants. Abbreviations used in (G) and $(\mathrm{H})$ : yc, young carpel before anthesis; mc, mature carpel at anthesis; $u$, untreated plants; pTRV2-E, plants treated with pTRV1 and pTRV2-E; pTRV2AG1, plants silenced for ESCaAG1; pTRV2-AG2, plants silenced for ESCAAG2; pTRV2-AG1/2, plants silenced for ESCaAG1 and ESCaAG2.

Table 2 Stamen numbers of in EScaAG1, EScaAG2, and EScaAG1/2 VIGS treated plants

\begin{tabular}{lllll}
\hline & $\begin{array}{l}\text { Untreated/pTRV1 and pTRV2- } \\
\text { E treated }\end{array}$ & $\begin{array}{l}\text { EScaAG1 VIGS } \\
\text { treated }\end{array}$ & $\begin{array}{l}\text { EScaAG2 VIGS } \\
\text { treated }\end{array}$ & $\begin{array}{l}\text { EScaAG1/2 VIGS } \\
\text { treated }\end{array}$ \\
\hline No. of flowers analyzed & 28 & 244 & 242 & 333 \\
\hline $\begin{array}{l}\text { No. of flowers without homeotic conversions } \\
\text { Average no. of stamens in flowers without }\end{array}$ & 28 & 93 & 123 & 92 \\
\hline \begin{tabular}{l} 
homeotic conversions \\
\hline
\end{tabular} & $26.2 \pm 1.9$ & $29^{*} \pm 4.1$ & $28.2^{*} \pm 2.9$ & $28.6^{*} \pm 4.5$ \\
\hline
\end{tabular}

* Significant change to untreated control plants (ANOVA test) 
leading to EScaAG2 [30]. A recent shift in constraint on EScaAG2 may not be detectable [32], but the molecular evolutionary analyses indicate that both EScaAG2 and EScaAG1 have been evolving under selective constraint for much of the approximately 50 million years since duplication. These results suggest that both EScaAG1 and 2 have been selectively maintained in the lineage leading to $E$. californica.

Gymnosperm and angiosperm $A G$ homologs are highly conserved but gene duplications have spurred functional diversification. The observation that knocking down EScaAG1 and 2 individually results in overlapping phenotypes can be explained by two alternative scenarios. First, the two poppy $A G$ paralogs may be working redundantly in the specification of floral organ identity and floral meristem determinacy. Alternatively, the VIGS method may not be able to individually silence paralogs with highly similar sequences. Our results are not fully consistent with either of these interpretations. Expression analyses of single knock down VIGS plants showed that transcript abundance of both genes was decreased, but EscaAG2 was silenced more strongly than EscaAG1. With respect to the first scenario, the selective maintenance of fully redundant genes over 50 million years is highly unlikely. Full knockouts (vs. knock downs) for each paralog may be required to reveal subtle functional divergence.

Differences in expression between EScaAG1 and EScaAG2 (Figure 2A) and deviations in spatial distribution of the homeotic conversions of stamens into petals (Figure 3I-K) hint at some degree of subfunctionalization. However, we were not able to relate the distinct phenotypes of only outer stamen whorl homeotic conversions in the case of EScaAG1 VIGS and only inner stamen whorl conversion in the EScaAG2-silenced flowers to the expression EScaAG1 and EScaAG2 expression data. In almost all analyzed floral buds we have simultaneous down-regulation of both genes with always a higher residual EScaAG1 expression than EScaAG2 expression, suggesting that subtle spatial expression difference at a very early developmental stage might play a role which we were not able to detect with our expression analysis. A less transient approach such as stable transformation with hairpin RNA constructs that would be able to silence EScaAG1 and EScaAG2 expression individually is required to rigorously characterize functional domains for ESCaAG1 and 2, and test the subfunctionalization hypothesis.

Another characteristic of the EScaAG1 and 2 VIGS phenotype is the loss of carpel organ identity. The most common phenotype observations were flattened green gynoecia or flat petaloid gynoecia showing an orange pigmentation and cell surface structure typical for petals (Figure 4A-E, Additional file 2.). The latter finding indicates that in E. californica, homeotic conversions of gynoecia into petaloid structures can occur when the $\mathrm{C}$ function is missing. This homeotic conversion coincides with the expansion of the expression domains of two class B genes, EScaDEF2 and EScaGLO, into the central floral whorl of EScaAG1 and EScaAG2 VIGS treated plants. The third B class gene, EScaDEF1 is also expressed in the gynoecia of untreated plants and expression levels are unaffected by reduction of $\mathrm{C}$ class gene expression in VIGS treated plants (Figure 5). These findings suggest that EScaDEF1 expression is independent of class $C$ gene expression while EScaDEF2 and EScaGLO are negatively regulated by class $C$ genes in the central floral whorl.

Interestingly, EScaDEF2 and EScaGLO are expressed in parallel with the class $C$ genes in the stamen whorl which indicates $C$ independent expression of the two class $B$ genes in stamen whorls in contrast to $C$ dependent expression in the central floral whorl. Thus, a cofactor $(\mathrm{X})$ restricted to the central whorl can be postulated to inhibit expression of EScaDEF2 and EScaGLO expression along with the $C$ class proteins EScaAG1 and EScaAG2 (Figure 5).

This type of $\mathrm{C}$-dependent regulation of $\mathrm{B}$ class genes is in contrast to the strong Arabidopsis ag-3 mutant, where full homeotic conversions of stamens into petals and carpels into sepals are observed. Even in the weaker ag-4 mutant, the carpel is not converted into a petal-like structure, but rather into a sepal [33]. Single or double mutants $\operatorname{sh} 1 / \operatorname{sh} p 2$ do not show any floral homeotic functions in Arabidopsis. Phenotypic effects are detectable only after fertilization [34]. In contrast, the Antirrhinum ple-1/far double mutant shows the type of floral homeotic conversions we observe in poppy: carpels are converted into petal-like structures and additional flower enclosed inside the fourth whorl unlike in the third whorl in Arabidopsis [15]. In the Arabidopsis ag mutant, the expression of the $\mathrm{B}$ function genes AP3 and $P I$ in the fourth whorl is prevented by the action of SUPERMAN (SUP) [35] and carpels are converted to sepal-like organs [36]. Therefore, it seems that the regulation of $B$ function genes is independent of $C$ class gene function in Arabidopsis. However, at this point we cannot exclude the hypothesis that $A G$ together with the closely related $S H P 1$ and $S H P 2$ genes work with SUP to repress B gene expression in the fourth whorl. In the Antirrhinum ple-1/far double mutant, an expansion of the $B$ function expression domain towards the fourth whorl was observed as a result of a $C$ function reduction. It was suggested that the putative SUP orthologs in Antirrhinum, OCTANDRA (OCT) requires PLE or FAR to exclude $B$ function gene expression from the fourth whorl while SUP in Arabidopsis acts independently of $A G$ [15]. 


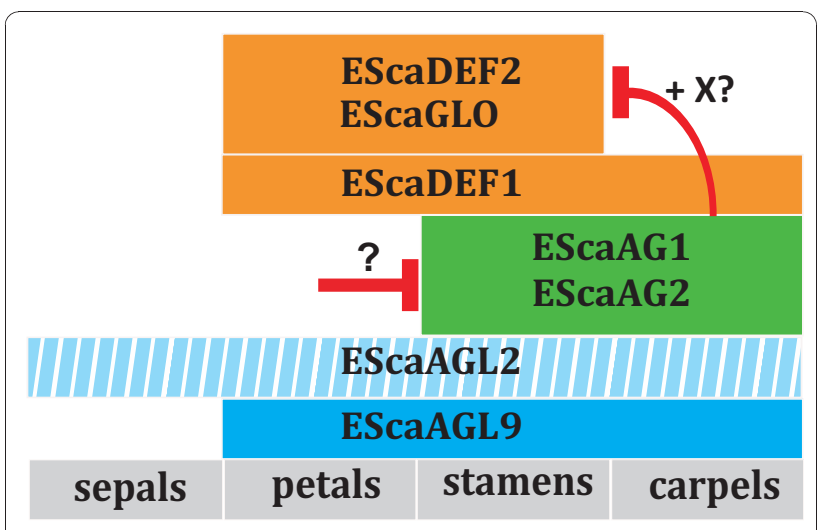

Figure 5 Hypothesis on the regulation of class $C$ dependent $B$ gene expression in $E$. californica. This modified BCE model of $E$. californica floral organ identity specification includes the B class genes (orange boxes) ESCaDEF1, ESCaDEF2, and ESCaGLO that are supposed to be expressed in second and third (stamen) whorl. Expression of ESCaDEF1 is also found in the central whorl. Two class $C$ genes (green boxes) are expressed in the stamen and central whorl, and the two class E genes ESCaAGL2 and ESCaAGL9 (blue boxes) are expressed in all whorls except for the sepal whorl for ESCaAGL9 [43] while no information is available on the expression domain of ESCaAGL2. Red bars indicate repression of gene expression. It remains unclear by which mechanism ESCaAG1 and EScaAG2 expression is restricted to the reproductive whorls of the flower and if repression of ESCaDEF2 and ESCaGLO by the two class $\mathrm{C}$ genes is direct or mediated by a co-factor. An A function has not yet been described in California poppy.

Our analysis of the EScaAG1 and EScaAG2 VIGS flowers suggests that the regulation of poppy $B$ function genes is more similar to Antirrhinum than to Arabidopsis because we also find an expansion of petal-like tissues specified by the B function in the fourth whorl. As postulated for Antirrhinum, the negative regulation of $\mathrm{B}$ function genes in the fourth whorl may involve the activation of an E. californica SUP ortholog. A poppy SUP ortholog could be positively regulated by EScaAG1 and 2 or interact with these genes to restrict $B$ function expression to the second and third whorl in wild type plants.

The fact that B gene expression is restricted by $\mathrm{C}$ function in E. californica as a representative of a basal eudicot lineage and Antirrhinum, a member of the asterid clade, in contrast to $\mathrm{C}$ independent regulation in Arabidopsis indicates that the former regulatory scenario might be ancestral. However, $\mathrm{C}$ function dependent regulation of $\mathrm{B}$ class genes has also not been reported in monocots such as rice. Down regulation of the rice $A G$ homolog, OsMADS58, did not result in expansion of the expression domains for B class genes and carpel to lodicule transformation have not been observed in osmads 3 mutants or OsMADS58 RNAi lines [17].

This suggests three possibilities for the evolution of class $C$ dependent regulation of class $B$ gene expression: (i) This type of regulation had evolved before the monocot and eudicot lineages diverged but was lost independently, in lineages leading to Arabidopsis and rice. (ii) The C-dependent regulation of B expression evolved once in the eudicots before the divergence of Ranunculales and was lost in the lineage leading to Arabidopsis after their split from the asterids. (iii) Class $\mathrm{C}$ genes were recruited twice independently, once in the lineage that led to E. californica after it diverged from the rest of the dicots and a second time in the lineage leading to Antirrhinum after its divergence from the lineage leading to Arabidopsis. Since class C floral homeotic mutants are not yet available from basal angiosperms or non-grass monocots all three of these scenarios are equally parsimonious.

As reported for Arabidopsis ag mutants, a reduction of EScaAG1 and 2 function in E. californica leads to defects in floral meristem termination, albeit in a more complex pattern than observed in Arabidopsis. The stamen whorls of ESCaAG1 and 2 VIGS-treated plants are more numerous than in the control plants even if the phenotype is mild, for example, no homeotic conversions of reproductive organs (Figure 3F). These observations support inferences drawn from work on $A$. thaliana and A. majus where mild reductions in C-function affect floral meristem determinacy $[37,38]$. The morphogenesis of E. californica flowers differs from most core eudicots, for example, Arabidopsis, in that the innermost stamen whorls are still being formed when the central gynoecium is initiated. A ring of cells with meristematic activity around the gynoecium is maintained while the central floral meristem is consumed in the process of gynoecium initiation [20]. This suggests that a mild reduction in EScaAG1 and 2 expression is sufficient for a prolonged meristem activity in this ring shaped meristem that produces additional stamen whorls in EScaAG1 and 2 VIGS-treated flowers. Additionally, our results suggest that EScaAG1 and 2 regulate the termination of meristem activity in E. californica. Regulation of meristematic activity was observed in the central floral meristem and the ring meristem that gives rise to stamen whorls independently of the ceasing central floral meristem activity (Table 2). The influence of EScaAG1 and 2 VIGS on the stamen whorls is especially interesting as stamen numbers in wild type $E$. californica are phenotypically variable, ranging from 18 up to 34 stamens when individuals are grown under identical conditions and constant light [20]. Even slight differences in the timing and dose of EScaAG1 and EScaAG2 transcript abundance between plants could account for these stamen number variations in wild type plants. The number of stamens in E. californica generally coincides with the plant's stature: as has been reported for Stellaria media (chickweed) [39], healthier plants produce 
more stamens. Our analyses suggest that the number of stamens produced is dependent on the amount of EScaAG1 and EScaAG2 transcript in E. californica flowers. This might indicate a stature-dependent regulation of class $\mathrm{C}$ floral homeotic genes in the ring-like meristem. Moreover, a direct link could exist between floral homeotic gene action and male fecundity in natural populations.

This additional function of the class $C$ genes in $E$. californica in the zone of meristematic activity around the gynoecium might represent a more general mode of function for class $C$ genes in the large subgroup of angiosperms with several stamen whorls and often varying stamen numbers. The duration of class $C$ genes activity in the meristems generating these reiterating stamen whorls might also determine stamen number in these species.

Our study on the function EScaAG1 and EScaAG2 in $E$. californica reveal that the VIGS method is suitable to analyze the evolution of gene regulation by enabling gene function analysis in non-model plants for which transgenic approaches are difficult to achieve. This work shows that gene function and the regulation of floral homeotic genes vary among plant lineages. Looking forward, the importance of VIGS for assessing gene function in non-model species will increase as advances in sequencing technologies result in full transcriptome and even genome sequences for an expanding number of species sampled across the plant tree of life. While sequence data will allow characterization of amino acid conservation and gene duplication events, functional studies in non-model species will be required to elucidate the evolution of regulatory networks influencing flowering time and floral form over angiosperm history.

\section{Materials and methods \\ Expression analysis \\ $Q-P C R$}

Q-PCR assays were performed on floral organs, leaves, young fruits, and buds of different developmental stages in wild type plants. For expression analysis of the EScaAG1 and EScaAG2 VIGS-treated plants, a single bud ( 1 to $2 \mathrm{~mm}$ in diameter) was examined. For the analysis of class C and B genes in VIGS-treated plants, single gynoecia of either buds of 5 to $8 \mathrm{~mm}$ diameter (young carpel) or from open flowers (mature carpel) were collected. All samples were analyzed in three technical replicates. One $\mu \mathrm{g}$ of total RNA was reverse transcribed into cDNA using random hexamer primers and the SuperScript III Kit (Invitrogen, Karlsruhe, Germany). A total of $5 \mu$ l of 1:50 diluted cDNA was used as a template. DEF1 and Actin primers were designed with the help of the UPL probe program (Roche, Mannheim, Germany); all other primers sets were designed with one intron spanning primer (primer details are in Additional file 3). Paralog specific primer pairs consist of forward primers spanning at least one intron and a reverse primer spanning the deletion part of EScaAG1 in 3' coding region were used to discriminate between EScaAG1 and EScaAG2 and the PCR product was sequenced to confirm primer specificity and the primer melting curves were analyzed. Eschscholzia Actin2 and GAPDH were used as reference genes. The Real-Time PCR reaction mix consisted of: $5 \mu \mathrm{l}$ of cDNA (1:50 dilution), $10 \mu \mathrm{l}$ of SYBR Green mix (Roche) and 0.8 to 1.2 pM primers. The UPL Real-Time PCR mix consisted of $5 \mu \mathrm{l}$ of 1:50 diluted cDNA, 100 nM UPL probe (Roche, \#132 for EScaDEF1 and \#136 for Actin) and 0.04 pM of each primer. Real-Time PCR was performed using a Light Cycler 480 (Roche) with the following cycle conditions: initial heating of $95^{\circ} \mathrm{C}$ for 5 minutes, and 45 cycles of $10 \mathrm{~s}$ at $95^{\circ} \mathrm{C}, 10 \mathrm{~s}$ at $60^{\circ} \mathrm{C}$ and $10 \mathrm{~s}$ at $72^{\circ} \mathrm{C}$. Cp values were analysed according to the Genorm manual and accurate normalization was carried out by geometric averaging of multiple internal control genes [40].

\section{In situ hybridisation}

Non-radioactive in situ hybridization followed essentially the protocol of [41]. The EscaAG1 and EScaAG2 coding regions were cloned into the pDrive vector (Qiagen, Hilde, Germany), the digoxigenin-labelled RNA probes were transcribed using SP6 polymerase (Roche) and subsequently hybridized to floral tissue sections.

\section{Virus-induced gene silencing}

A 395 bp fragment of EScaAG1 was amplified from the EScaAG1 coding region by using the primers VIGSEcAG1A to add a $B a m H I$ restriction site to the 5 ' end of the PCR product and EcAG1VIGS to add an XhoI restriction site to the 3 ' end (primer sequences reported in Additional file 3). The amplicon was digested with BamHI and XhoI and cloned into a similarly cut pTRV2 vector [42]. A 477 bp fragment of EScaAG2 was amplified from the EScaAG2 coding region by using the primers VIGSEcAG2A to add a $B a m H I$ restriction site to the 5' end of the PCR product and EcAG2VIGS to add an $X h o$ I restriction site to the 3' end. The amplicon was digested with $\mathrm{BamHI}$ and $X h o \mathrm{I}$ and cloned into a similarly cut pTRV2. pTRV2-EScaAG1/AG2 was constructed by a $190 \mathrm{bp}$ fragment of EScaAG1 was amplified from the EScaAG1 coding region by using the primers XbaVIGSEcAG1Bfw to add a XbaI restriction site to the 5' end of the PCR product and EcAG1VIGSXhorev to add a XhoI restriction site to the 3' end. The amplicon was digested with XbaI and XhoI. A 214 bp fragment of EScaAG2 was amplified from the EScaAG2 coding region by using the primers EcoVIGSEcAG2Afw to add an EcoRI restriction site to the 5' end of the PCR product and EcAG2VIGSXbarev to add an XbaI restriction site to the 3' end. The amplicon was digested with 
EcoRI and $X b a \mathrm{I}$ and was then ligated together with the EScaAG1 fragment into the EcoRI and XhoI cut pTRV2 vector producing the pTRV2-EScaAG1/AG2 plasmid. The vector inserts of the double construct were confirmed by restriction digestion and sequencing. The resulting plasmids were sequenced and transformed into Agrobacterium tumefaciens strain GV3101. The agroinoculation was performed by injecting the Agrobacterium suspension into the shoot apical meristem as described by [22].

\section{Scanning electron microscopy and histology}

Gynoecia of EScaAG1 and EScaAG2 VIGS-treated and untreated plants were analyzed by Scanning Electron Microscopy [14] for changes in the cell surface structure. The gynoecia were incubated in $100 \%$ methanol for 10 minutes and subsequently for 10 minutes in $100 \%$ ethanol. Then they were kept overnight at room temperature in $100 \%$ ethanol and dried with a Critical Point Dryer, gold coated, and examined under the SEM (ISI-100B, International Scientific Instruments, Pleasanton, CA, USA). First formed buds of 1.6 to $2.5 \mathrm{~mm}$ in diameter were collected for histological analysis and stained with Safranin and Fast Green as described by [22].

\section{Additional material}

Additional file 1: Supplemental Figure 1: Alignment of the EScaAG1 and EScaAG2 protein sequences. Amino acids identical between two paralogs are indicated by dots; dashes indicate deletion of five amino acids located in the C-terminal region of ESCaAG2. Dissimilar residues are indicated by the respective amino acids.

Additional file 2: Supplemental Figure 2: Phenotypes observed in the gynoecium of ESCaAG VIGS treated plants. The Y-axis denotes the percentages of different carpel identity phenotypes obtained by VIGS (pTRV2-ESCaAG1, $\mathrm{n}=239$; ESCaAG2, $\mathrm{n}=209$, ESCaAG1/2, $\mathrm{n}=261$ flowers). Differently treated VIGS plants are shown on the X-axis. The green color indicates the occurrence of flat green gynoecia; the orange color symbolizes flat orange gynoecia. Stripes indicate gynoecia enclosing ovules, plane color indicates a gynoecium lacking ovules, and the dotted pattern indicates additional organs enclosed by the gynoecium.

Additional file 3: Supplemental Table 1: Sequences of primers used in this study.

\section{Abbreviations}

AG: the floral homeotic $C$ function gene AGAMOUS of A. thaliana; AP3: the floral homeotic B function gene APETALA3 of A. thaliana; ESCAAG1: $E$. californica ortholog of $A G$; ESCaAG2: E. californica ortholog of $A G$; EScaAGL 11: E. californica ortholog of the the ovule specific gene SEEDSTICK (formerly known as AGL11) of A. thaliana; EScaDEF1: E. californica ortholog of the A. majus floral homeotic $B$ function gene DEFICIENS; ESCaDEF2: E. californica ortholog of the A. majus floral homeotic B function gene DEFICIENS; EScaGLO: E. californica ortholog of the A. majus floral homeotic B function gene GLOBOSA; FAR: floral homeotic C function gene FARINELLI in A. majus: OCT: putative stamen and carpel boundary specifying gene OCTANDRA in A. majus; OSMADS3: floral homeotic C function gene of O. sativa; OSMADS58: floral homeotic $C$ function gene of $O$. sativa; PI: the floral homeotic B function gene PISTILLATA of A. thaliana; PLE: the A. majus floral homeotic C function gene PLENA; Q RT-PCR: Quantitative Reverse Transcriptase polymerase chain reaction; SEM: Scanning electron microscopy; SHP: the
}

SHATTERPROOF gene of A. thaliana; SUP: the stamen and carpel boundary specifying gene SUPERMAN of A. thaliana; TRV: Tobacco rattle virus; UPL: Universal probe library; UTR: Untranslated region; VIGS: Virus induced gene silencing; ZAG1: floral homeotic C function gene of Z. mays; ZMM2: floral homeotic $C$ function gene $Z$. mays.

\section{Acknowledgements}

We thank Zsuzsanna Schwarz-Sommer as well as David Smyth for valuable discussions about the VIGS phenotypes and S.P. Dinesh-Kumar for providing the pTRV vectors. We thank the Becker lab members for lively discussions, Teja Shidore and Gitanjali Darmadhikari for their help with the phenotyping. We also thank Werner Vogel and Angelika Trambacz for poppy plant growth and care. This work was made possible with funding from the German Research Foundation (DFG) grant to A. B. (BE 2547/6-1, 6-2) and funding by the University of Bremen. This paper is dedicated to the memory of the late Zsuzsanna Schwarz-Sommer whose interest in this project was warmly appreciated, and whose contribution to the field was inspirational.

\section{Author details}

${ }^{1}$ University of Bremen, Fachbereich 02 Biology/Chemistry, Evolutionary Developmental Genetics Group Leobener Str., UFT, 28359 Bremen, Germany. ${ }^{2}$ Department of Plant Biology, University of Georgia, Athens, GA 30602-7271, USA.

\section{Authors' contributions}

AYL participated in the expression analysis and phenotype characterization. $\mathrm{SL}$ carried out the cloning work and participated in expression analysis and phenotype characterization. SO carried out and interpreted the in situ hybridizations. RE analyzed and interpreted the Real-Time PCR data. JLM performed sequence divergence time estimates and molecular evolutionary analyses and edited the manuscript. AB conceived of the study, participated in its design and coordination. AYL and $A B$ wrote this manuscript. All authors read and commented on drafts of the manuscript and approved the final manuscript.

\section{Competing interests}

The authors declare that they have no competing interests.

Received: 7 May 2010 Accepted: 1 December 2010

Published: 1 December 2010

\section{References}

1. Fletcher JC: Shoot and floral meristem maintenance in Arabidopsis. Annual Review of Plant Biology 2002, 53:45-66.

2. Coen ES, Meyerowitz EM: The war of the whorls: genetic interactions controlling flower development. Nature 1991, 353:31-37.

3. Theissen G, Saedler H: Floral quartets. Nature 2001, 409:469-471.

4. Bowman JL, Drews GN, Meyerowitz EM: Expression of the Arabidopsis floral homeotic gene AGAMOUS is restricted to specific cell types late in flower development. Plant Cell 1991, 3:749-758.

5. Bowman JL, Smyth DR, Meyerowitz EM: Genes directing flower development in Arabidopsis. The Plant Cell 1989, 1:37-52.

6. Rutledge R, Regan R, Nicolas O, Fobert P, Côté C, Bosnich W, Kauffeldt C, Sunohara G, Séguin A, Stewart D: Characterization of an AGAMOUS homologue from the conifer black spruce that produces floral homeotic conversions when expressed in Arabidopsis. The Plant Journal 1998, 15:625-634.

7. Winter K-U, Becker A, Munster T, Kim JT, Saedler H, Theissen G: MADS-box genes reveal that gnetophytes are more closely related to conifers than to flowering plants. Proceedings of the National Academy of Sciences 1999, 96:7342-7347.

8. Jager M, Hassanin A, Manuel M, Guyader HL, Deutsch J: MADS-Box Genes in Ginkgo biloba and the Evolution of the AGAMOUS Family. Mol Biol Evol 2003, 20:842-854.

9. Becker A, Theißen G: The major clades of MADS-box genes and their role in the development and evolution of flowering plants. Molecular Phylogenetics and Evolution 2003, 29:464-489.

10. Kramer EM, Jaramillo MA, Di Stilio VS: Patterns of Gene duplication and functional evolution during the diversification of the AGAMOUS subfamily of MADS box genes in angiosperms. Genetics 2004, 166:1011-1023. 
11. Irish VF: The evolution of floral homeotic gene function. BioEssays 2003, 25:637-646.

12. Liljegren SJ, Ditta GS, Eshed Y, Savidge B, Bowman JL, Yanofsky MF: SHATTERPROOF MADS-box genes control seed dispersal in Arabidopsis. Nature 2000, 404:766-770.

13. Colombo M, Brambilla V, Marcheselli R, Caporali E, Kater MM, Colombo L: A new role for the SHATTERPROOF genes during Arabidopsis gynoecium development. Developmental Biology 2010, 337:294-302.

14. Bradley D, Carpenter R, Sommer H, Hartley N, Coen E: Complementary floral homeotic phenotypes result from opposite orientations of a transposon at the plena locus of Antirrhinum. Cell 1993, 72:85-95.

15. Davies B, Motte P, Keck E, Saedler H, Sommer H, Schwarz-Sommer Z: PLENA and FARINELLI: redundancy and regulatory interactions between two Antirrhinum MADS-box factors controlling flower development. EMBO J 1999, 18:4023-4034

16. Mena M, Ambrose BA, Meeley RB, Briggs SP, Yanofsky MF, Schmidt RJ Diversification of C-function activity in maize flower development. Science 1996, 274:1537-1540.

17. Yamaguchi T, Lee DY, Miyao A, Hirochika H, An G, Hirano H-Y: Functional Diversification of the two c-class MADS box genes OSMADS3 and OSMADS58 in Oryza sativa. Plant Cell 2006, 18:15-28.

18. Zahn LM, Leebens-Mack JH, Arrington JM, Hu Y, Landherr LL, dePamphilis CW, Becker A, Theissen G, Ma H: Conservation and divergence in the AGAMOUS subfamily of MADS-box genes: evidence of independent sub- and neofunctionalization events. Evolution \& Development 2006, 8:30-45.

19. Drews NG, Bowman JL, Meyerowitz EM: Negative regulation of the Arabidopsis homeotic gene AGAMOUS by the APETALA2 product. Science 1991, 65:991-1002.

20. Becker A, Gleissberg S, Smyth DR: Floral and vegetative morphogenesis in California poppy (Eschscholzia californica CHAM.). International Journal of Plant Sciences 2005, 166:537-555.

21. Wege S, Scholz A, Gleissberg S, Becker A: Highly efficient virus-induced gene silencing (VIGS) in California poppy (Eschscholzia californica): An evaluation of VIGS as a strategy to obtain functional data from nonmodel plants. Ann Bot 2007, 100:641-649.

22. Orashakova S, Lange M, Lange $S$, Wege S, Becker A: The CRABS CLAW ortholog from California poppy (Eschscholzia californica, Papaveraceae), EcCRC, is involved in floral meristem termination, gynoecium differentiation and ovule initiation. Plant J 2009, 58:682-93.

23. McGonigle B, Bouhidel K, Irish VF: Nuclear localization of the Arabidopsis APETALA3 and PISTILLATA homeotic gene products depends on their simultaneous expression. Genes Dev 1996, 10:1812-1821.

24. Zahn LM, Leebens-Mack J, DePamphilis CW, Ma H, Theissen G: To B or Not to $B$ a flower: the role of DEFICIENS and GLOBOSA orthologs in the evolution of the angiosperms. J Hered 2005, 96:225-240.

25. Force A, Lynch M, Pickett FB, Amores A, Yan Y-I, Postlethwait J: Preservation of duplicate genes by complementary, degenerative mutations. Genetics 1999, 151:1531-1545.

26. Nowak MA, Boerlijst MC, Cooke J, Smith JM: Evolution of genetic redundancy. Nature 1997, 388:167-171.

27. Wagner A: Redundant gene functions and natural selection. Journal of Evolutionary Biology 1999, 12:1-16.

28. Cui L, Wall PK, Leebens-Mack JH, Lindsay BG, Soltis DE, Doyle JJ, Soltis PS, Carlson JE, Arumuganathan K, Barakat A, Albert VA, Ma H, dePamphilis CW: Widespread genome duplications throughout the history of flowering plants. Genome Res 2006, 16:738-749.

29. Sanderson MJ: r8s: inferring absolute rates of molecular evolution and divergence times in the absence of a molecular clock. Bioinformatics 2003, 19:301-302.

30. Shan $\mathrm{H}$, Zahn L, Guindon S, Wall PK, Kong H, Ma H, dePamphilis CW, Leebens-Mack J: Evolution of plant MADS box transcription factors: evidence for shifts in selection associated with early angiosperm diversification and concerted gene duplications. Mol Biol Evol 2009, 26:2229-2244.

31. Moore MJ, Bell CD, Soltis PS, Soltis DE: Using plastid genome-scale data to resolve enigmatic relationships among basal angiosperms. Proceedings of the National Academy of Sciences 2007, 104:19363-19368.

32. Leebens-Mack J, dePamphilis C: Power analysis of tests for loss of selective constraint in cave crayfish and nonphotosynthetic plant lineages. Mol Biol Evol 2002, 19:1292-1302.
33. Sieburth $L E$, Running MP, Meyerowitz EM: Genetic separation of third and fourth whorl functions of AGAMOUS. Plant Cell 1995, 7:1249-1258.

34. Liljegren SJ, Ditta GS, Eshed Y, Savidge B, Bowman JL, Yanofsky MF: SHATTERPROOF MADS-box genes control seed dispersal in Arabidopsis. Nature 2000, 404:766-770.

35. Sakai H, Medrano LJ, Meyerowitz EM: Role of SUPERMAN in maintaining Arabidopsis floral whorl boundaries. Nature 1995, 378:199-203.

36. Jack T, Brockman LL, Meyerowitz EM: The homeotic gene APETALA3 of Arabidopsis thaliana encodes a MADS box and is expressed in petals and stamens. Cell 1992, 68:683-697.

37. Mizukami Y, Ma H: Separation of AG function in floral meristem determinacy from that in reproductive organ identity by expressing antisense AG RNA. Plant Molecular Biology 1995, 28:767-784.

38. Causier B, Bradley D, Cook H, Davies B: Conserved intragenic elements were critical for the evolution of the floral C-function. The Plant Journal 2009, 58:41-52.

39. Haskell G: Variation in the number of stamens in the common chickweed. Journal of Genetics 1949, 49:291-301.

40. Vandesompele J, De Preter K, Pattyn F, Poppe B, Van Roy N, De Paepe A, Speleman F: Accurate normalization of real-time quantitative RT-PCR data by geometric averaging of multiple internal control genes. Genome Biol 2002, 3:RESEARCH0034.

41. Groot EP, Sinha N, Gleissberg S: Expression patterns of STM-like KNOX and Histone $\mathrm{H} 4$ genes in shoot development of the dissected-leaved basal eudicot plants Chelidonium majus and Eschscholzia californica (Papaveraceae). Plant Molecular Biology 2005, 58:317-331.

42. Ratcliff F, Martin-Hernandez AM, Baulcombe DC: Tobacco rattle virus as a vector for analysis of gene function by silencing. Plant Journal 2001, 25:237-245.

43. Zahn LM, Kong H, Leebens-Mack JH, Kim S, Soltis PS, Landherr LL, Soltis DE, Depamphilis CW, Ma H: The evolution of the SEPALLATA subfamily of MADS-box genes: a preangiosperm origin with multiple duplications throughout angiosperm history. Genetics 2005, 169:2209-2223.

doi:10.1186/2041-9139-1-13

Cite this article as: Yellina et al.: Floral homeotic $C$ function genes repress specific $B$ function genes in the carpel whorl of the basal eudicot California poppy (Eschscholzia californica). EvoDevo 2010 1:13.

\section{Submit your next manuscript to BioMed Central and take full advantage of:}

- Convenient online submission

- Thorough peer review

- No space constraints or color figure charges

- Immediate publication on acceptance

- Inclusion in PubMed, CAS, Scopus and Google Scholar

- Research which is freely available for redistribution

Submit your manuscript at www.biomedcentral.com/submit
C Biomed Central 